MATEC Web of Conferences 51, 02009 (2016)

DOI: $10.1051 /$ matecconf/20165102009

(C) Owned by the authors, published by EDP Sciences, 2016

\title{
Level-Ground Walking for 3D Quasi-Passive Walker with Flat Feet - Lateral-plane Input using McKibben-Type Artificial Muscle -
}

\author{
Akihiro Yamamoto ${ }^{1}$, Shinsaku Fujimoto ${ }^{1, a}$ and Tetsuya Kinugasa ${ }^{1}$ \\ ${ }^{1}$ Okayama University of Science, Engineering Graduate Course \\ 1-1 Ridai-cho, Kita-ku, Okayama, Japan
}

\begin{abstract}
Currently, many bipedal robots have been proposed to realize the high energy efficiency walking. The passive dynamic walking does not require control input. Generally, a foot of passive dynamic walking robot is an arc foot. In this paper, it is intended to establish a control method and control mechanism to achieve energy efficient and stable gate. Therefore, we developed 3D quasi-passive walker with flat feet driven by an antagonistic pneumatic artificial muscle. An antagonistic mechanism is constituted by a pair of McKibben muscle. And an antagonistic pneumatic system is used as joint actuators of linkage mechanisms which control the torque, joint stiffness and position simultaneously. Finally, this report shows that the 3D quasi-passive walking in the level ground can realize by the swinging (simple) input of the frontal direction, and the stride of the robot is proportional to lateral-plane input.
\end{abstract}

\section{Introduction}

An energy efficiency and locomotion such as human [1] are very important issues in a biped robot walking. Recently, a passive dynamic walking has received considerable research attention. The investigation of passive dynamic mechanism can help to solve both issues. The idea of passive dynamic walking was pioneered by McGeer [2]-[4] who studied the so-called compass gait biped, a two DOF walker that can achieve passive dynamic walking down shallow inclines powered only by gravity. Most previous studies of passive dynamic walker with arc-shaped feet (no feet) have been constrained by the locomotion of the sagittal plane [5][6]. Since the ground-direction contact occurs only at a point, the arc-shaped feet provide insufficient friction against ground. Passive walkers with arc-shaped feet are unstable in the upright state.

Wisse et al.[7]-[9] studied a 2D passive walker with flat feet and ankle springs by utilizing the equivalence between the torsional spring and the arc-shaped feet. Therefore, the arc-shaped feet can be replaced by flat feet that are mounted on ankles with torsional spring stiffness.

In this paper, we develop the thee-dimensional quasi-passive walker with flat feet which is driven by antagonistic pneumatic artificial muscle.

This paper is organized as follows. Chapter 2 explains the principle of 3D quasi-passive walking, and antagonism pneumatic artificial muscle. Chapter 3 then presents the structure of quasi-passive

\footnotetext{
${ }^{a}$ Corresponding author : fuji@are.ous.ac.jp
} 
dynamic walker, followed by the method of our experiment and its results in Chapter 4. Finally, the paper concludes with the results of the 3D quasi-passive walking in the level ground.

\section{Relations of Arc foot and Equivalent spring constant}

Wisse et al. showed that the rigid arc-shaped feet can be replaced by flat feet that are mounted on ankles with a torsional spring stiffness. The spring stiffness has a similar effect as the foot radius; it reduces the sensitivity to disturbances due to friction (slipping) and collisions, and thus improves the disturbance handling. Therefore, 3D passive dynamic walking with flat feet are walking forms that extend the lateral motion to 2D passive dynamic walking in sagittal plane as indicated in Figure 1. They studied a 2D passive walker with flat feet and ankle springs by utilizing the equivalence between the torsional spring and the arc-shaped feet.

A typical antagonistic pneumatic mechanism consists of a pair of McKibben artificial muscles [10] as shown in Figure 2. The developed walker [11] has the equivalent characteristic of torsional spring to the walking robot by equipping antagonism pneumatic artificial muscle. It is confirmed that sustainable 3D quasi-passive walker can walk on a slope. In this study, we measure the natural frequency, the ankle rigidity and the stride of the walking robot based on 3D quasi-passive gait on the slope.

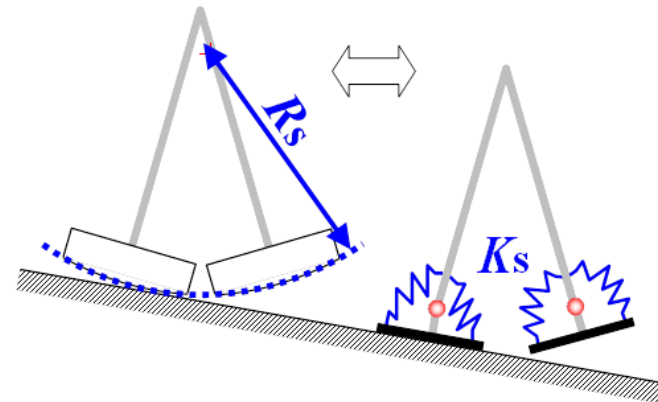

(a) Sagittal plane

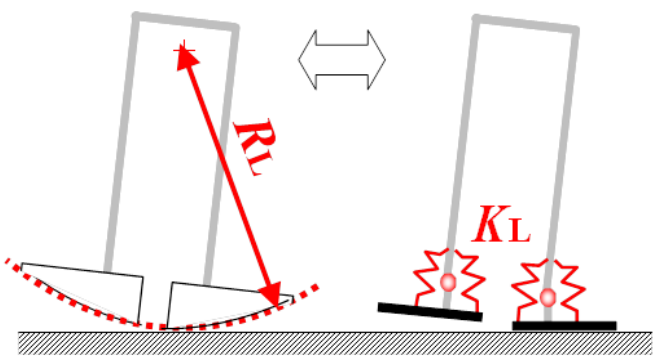

(b) Lateral plane

Figure 1. 3D passive dynamic walking with flat feet.
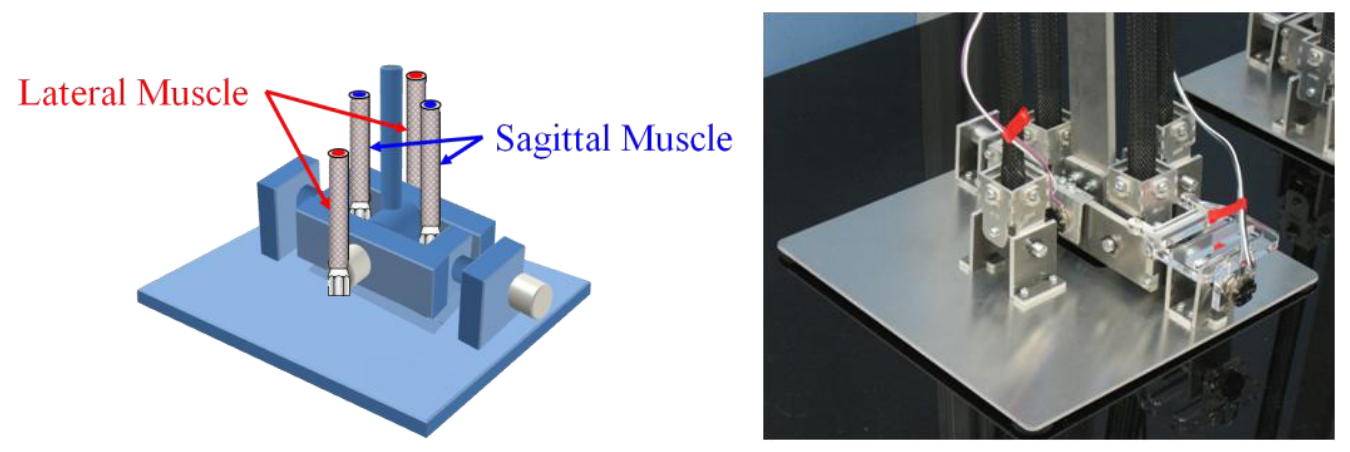

Figure 2. Structure of ankle joint.

\section{Structure of Bipedal Robot}

Figure 2 and Figure 3 showed whole structure and dimensions of the quasi-passive dynamic walker (TENBU 3). TENBU produced in this study is equipped the McKibben artificial muscle at sagittal and lateral direction of the ankle joint. The biped walking robot is equipped with micro-computer 
(Renesas Co. Ltd.,H8/3664), battery, air tank, On/Off valves and wireless module(ZigBee). Passive dynamic walking is a gait form that depends on the dynamic characteristics of biped walking robot. It is desirable that there is no external cable(umbilical cable) as much as possible. Thus, "TENBU" robot is a self-contained robot. The trial number of the walking experiment in level ground is about 5 times by using two air tanks(air capacity : 4 liter). Circular rubber sheet with the force sensor is attached to the soles of the feet to increase friction on the ground.

The micro-computer adjusts the internal pressure of artificial muscle by controlling the On/Off valve. The embedded controller that is the micro-computer gets the output voltage from six potentiometers and eight force sensors. Then, the sampling period of the On/Off valve control was about $30 \mathrm{~ms}$. However, the period of the sampling data is $70 \mathrm{~ms}$ by using the wireless ZigBee module(57600 bps).

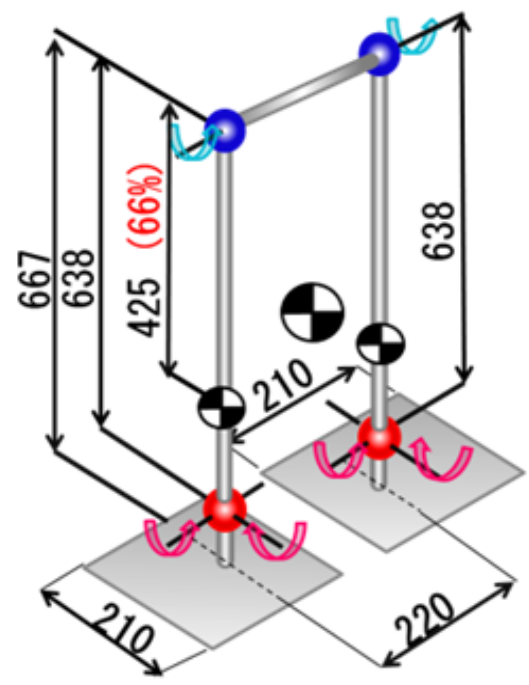

Figure 3. Structure and size of quasi-passive dynamic walker.

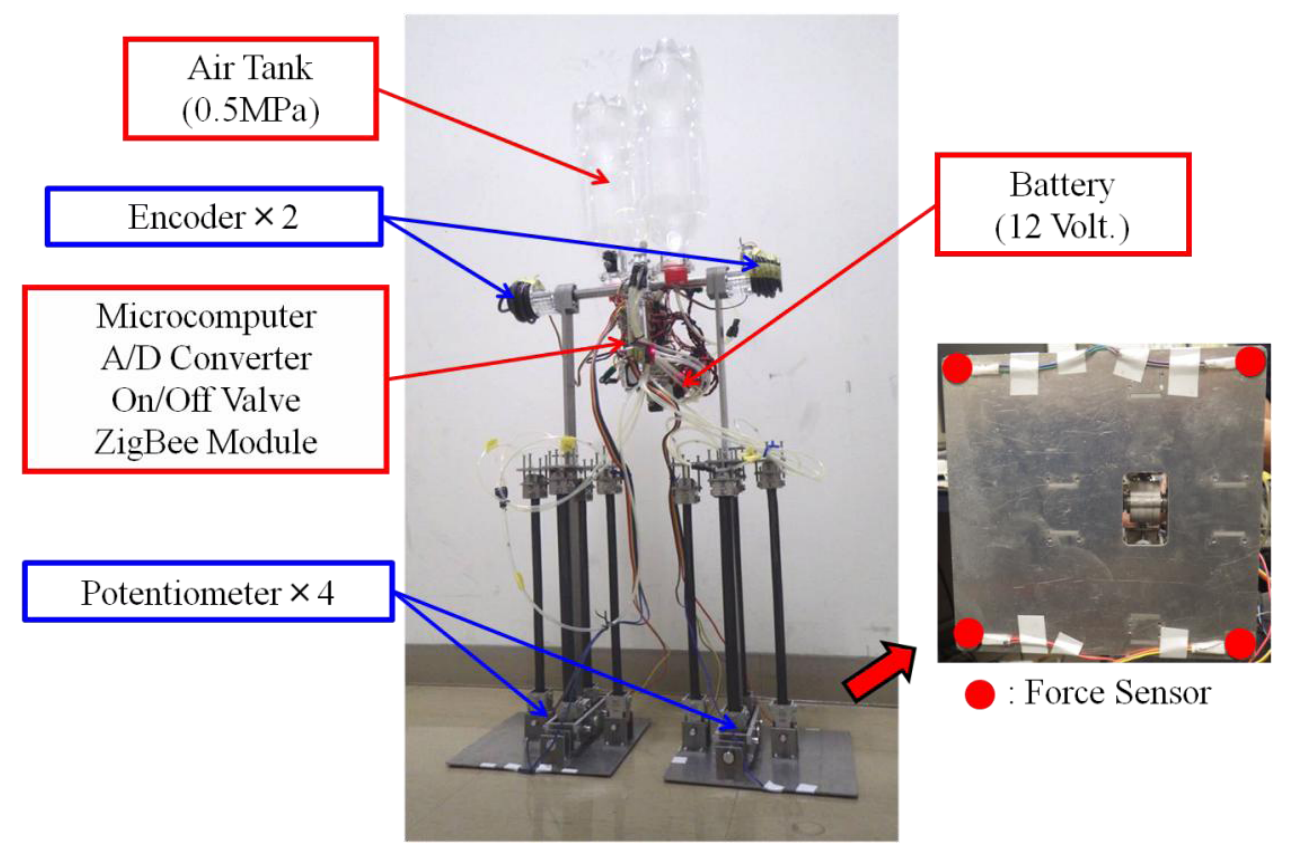

Figure 4. Quasi-passive dynamic walker (TENBU 3). 


\section{The experiment of 3D quasi-passive walking}

In this chapter, we carried out the experiment of 3D quasi-passive walking on the slope to confirm the effectiveness of the produced ankle experimentally and to acquire basic walking experiment in the level ground by lateral-plane input based on the walking data in the slope.

\subsection{Quasi-passive walking on the slope}

In this paragraph, we state the 3D quasi-passive walking experiment on the slop. Figure 5 shows the most common experimental result in which the $3 \mathrm{D}$ quasi-passive walker walked on the slope.

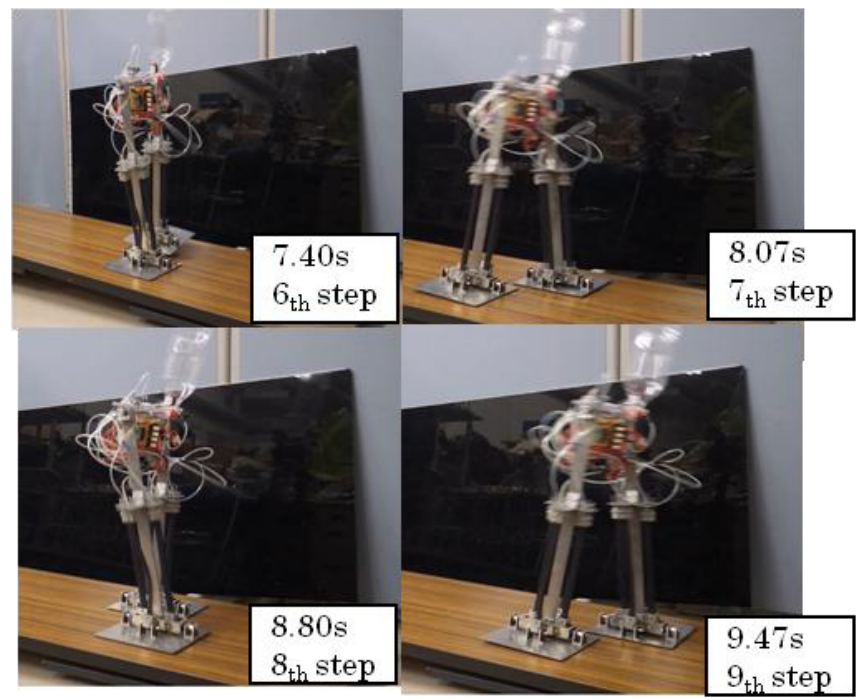

Figure 5. 3D quasi-passive walking on the slope.

The experimental conditions of the passive walking are as follows. But, valve controller is only control to keep the internal pressure of the McKibben artificial muscles to a constant value to maintain ankle rigidity constantly.

1. Angle of inclination of the slope : $4.0[\mathrm{deg}$.]

2. Internal pressure of artificial muscles : Sagittal plane direction : $0.20[\mathrm{MPa}]$

Lateral plane direction : $0.15[\mathrm{MPa}]$

As results of 3D quasi-passive walking experiment, the following gait data were obtained.

The sustained walking are possible under this condition:

1. Average step : $149[\mathrm{~mm}]$

2. Average walking period : $1.36[\mathrm{~s}]$ (Half period : $0.68[\mathrm{~s}]$ )

3. Average walking speed : $220[\mathrm{~mm} / \mathrm{s}]$

\subsection{The experiment of 3D quasi-passive walking in the level ground}

One of the purposes of this study includes that they realize 3D quasi-passive walking in the level ground. Therefore, we examined level ground walk by swing motion input (Lateral plane direction) using the quasi-passive walking without the knee as the first step in this paper.

We let fluctuate internal pressure $P_{\mathrm{R}}$ and $P_{\mathrm{L}}$ of the antagonism pneumatic artificial muscle attached to lateral plane direction of each ankle joint as following equation.

$$
\begin{array}{ll}
P_{R}=P_{A M P} \sin (\omega t)+P_{0} & :[\mathrm{Pa}](\text { Right }) \\
P_{L}=P_{A M P} \sin (\omega t-\pi)+P_{0} & :[\mathrm{Pa}](\mathrm{Left})
\end{array}
$$


where $P_{\text {AMP }}$ is amplitude, $\omega$ is angular frequency and $P_{0}$ is initial pressure. The initial pressure set up the sagittal plane muscle $0.20 \mathrm{MPa}$ and lateral plane muscle $0.15 \mathrm{MPa}$ from the experimental results of the passive walking on the slope. The angular frequency $\omega$ is set approximately $1.36 \mathrm{~s}$ (Half period : $0.68 \mathrm{~s}$ ) like the walking period of the $3 \mathrm{D}$ quasi-passive walking on the slope. In addition, the slope is inclined approximately 4 degrees.

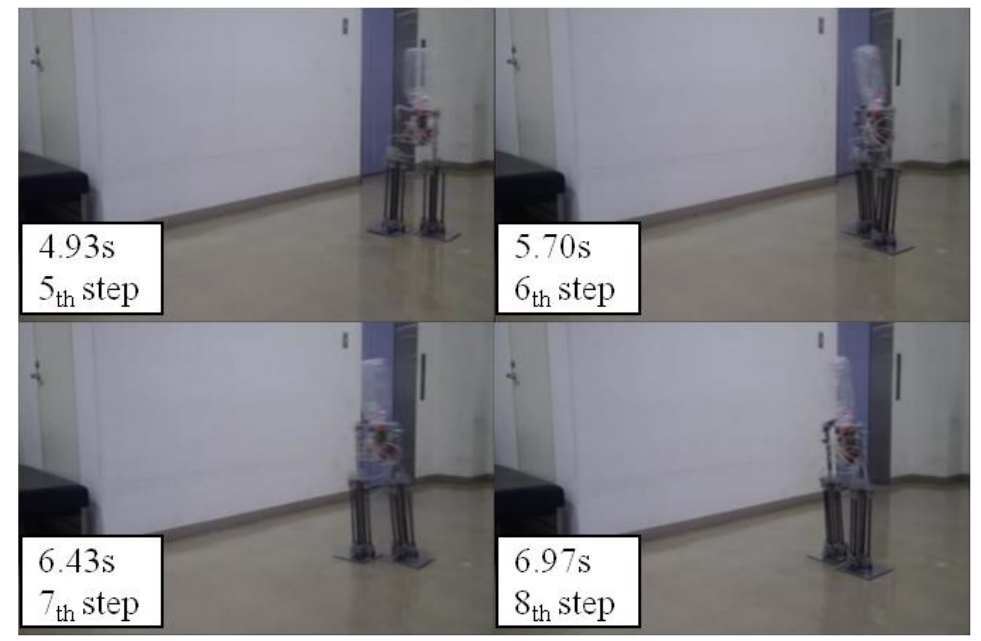

Figure 6. 3D quasi-passive dynamic walking (Level-ground).

Figure 6 shows the most common experimental results in which 3D quasi-passive walking in the level ground. TENBU was able to realize 3D quasi-passive walking in the level ground though it was only gave very simple lateral-plane input.

Figure 7 shows the relations between the stride and every half period. Half period that TENBU can walk from $0.3 \mathrm{~s}$ to $0.5 \mathrm{~s}$. In addition, we obtained the following equation that approximates to the liner function by the least-squares method from Figure 6 .

$$
b=320 \cdot T_{P}-70.4[\mathrm{~mm}]
$$

where $b$ is stride, $T_{\mathrm{P}}[\mathrm{s}]$ is half period. Therefore, it is thought that the stride and the period of lateral-plane inputs is proportion.

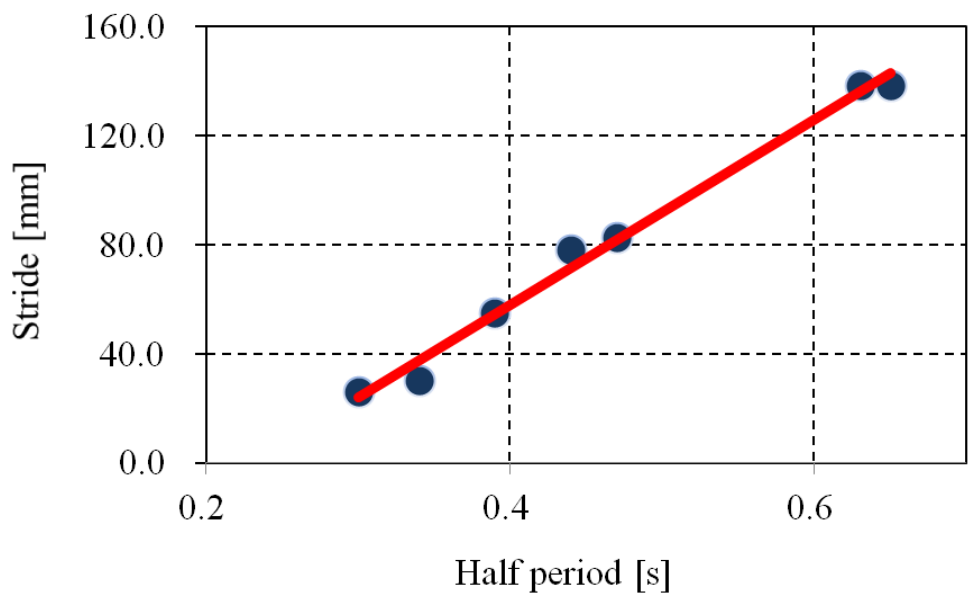

Figure 7. Relation between stride and half period. 


\section{Conclusions and Future Work}

This paper designed and produced the bipedal robot which had the antagonism pneumatic artificial muscle with flat feet. And we carried out 3D quasi-passive walking experiment. In addition, we carried out 3D quasi-passive walking experiment in the level ground by simple lateral-plane input. As a result, we were able to get the resulting knowledge.

1. The quasi-passive dynamic walker was able to be realized 3D quasi-passive walking in the level ground by means of giving simple lateral-plane input.

2. The proportion relations between the half period of lateral-plane input and the walking stride were discovered.

As the future work, we are going to conduct the gait analysis based on COP (Center Of Pressure) ROS (Roll Over Shapes) using encoder, potentiometer and power sensor.

\section{References}

[1] S. Collins, A. Ruina, R. Tedrake and M. Wisse : Efficient Bipedal Robots Based on PassiveDynamic Walkers, Science, 307, 1082-1085 (2005)

[2] T. McGeer : Passive Dynamic Walking :Simson Fraser University Burnaby British Columbia, CSS-ISS TR88-02 (Technical report), 1-51 (1988)

[3] T. McGeer : Passive Dynamic Walking, IJRR, 9, 62-82 (1990)

[4] T. McGeer : Passive Walking with Knees, Proceedings of the IEEE International Conference on Robotics and Automation, 3, 1640-1645 (1990)

[5] A. Goswami, B.Thuilot and B. Espiau : A study of the passive gait of a compass-like biped robot symmetry and chaos, IJRR, 17, 1282-1301 (1998)

[6] M. Garcia, A. Chatterjee, A. Ruina and M. Coleman : The Simplest Walking Model: Stability, Complexity, and Scaling, J BIOMECH ENG-T ASME, 120,281-288 (1998)

[7] M. Wisse at all : Ankle springs instead of arc-shaped feet for passive dynamic walkers, IEEERAS International Conference on Humanoid Robots, 110-116 (2006)

[8] T. Kinugasa, K. Yoshida, K. Kotake, K. Fujimura, H. Tanaka and K. Ogawa : 3D Passive Walker with Ankle Springs and Flat Feet, JRSJ, 27, 91-94 (2009) (in Japanese)

[9] T. Narukawa, K. Yokoyama, M. Takahashi and K. Yoshida : Design and Construction of a Simple 3D Straight-Legged Passive Walker with Flat Feet and Ankle Springs, JSDD, 3, 1 (2009)

[10] S. Fujimoto, T. Ono, K. Ohsaka and Z. Zhao : Modeling of Artificial Actuator and Control Design for Antagonistic Drive System, Transactions of JSME (C), 73 , no.730, 1777-1785 (2007) (in Japanese)

[11] S.Fujimoto, T.Kinugasa K.Yoshida and H.Watanabe: 3D Quasi-passive Walking of Bipedal Robot with Flat Feet -Quasi-passive Walker Driven by Antagonistic Pneumatic Artificial Muscle-, IJAMechS, 5, 95-104 (2013) 